\title{
A Portuguese isolate of Borrelia lusitaniae induces disease in $\mathrm{C} 3 \mathrm{H} / \mathrm{HeN}$ mice
}

\author{
NORDIN S. ZEIDNER, MARIA S. NÚNCIO*, BRADLEY S. SCHNEIDER, LISE GERN†, \\ JOSEPH PIESMAN, OTILIA BRANDÃO $\ddagger$ and ARMINDO R. FILIPE*
}

Centers for Disease Control and Prevention, Division of Vector-Borne Infectious Diseases, Fort Collins, CO 80522, USA, *Instituto Nacional de Saúde, Dr. Ricardo Jorge, Centro de Estudos de Vectores e Doencas Infecciosas, Águas de Moura, Portugal, †Institut de Zoologie, University of Neuchâtel, Neuchâtel, Switzerland and $\$$ Department of Pathology, São Bernardo Hospital, Setúbal, Portugal

\begin{abstract}
A low-passage, Portuguese isolate of Borrelia lusitaniae, strain PotiB2, was inoculated into $\mathrm{C} 3 \mathrm{H} / \mathrm{HeN}$ mice and disease was monitored by histopathology at 8 weeks after spirochaete challenge. Ear, heart, bladder, femoro-tibial joint, brain and spinal cord were examined. B. lusitaniae strain PotiB2 (6 of 10 mice) and B. burgdorferi sensu stricto strain N40 (9 of 10 mice) induced similar lesions in the bladder of infected mice characterised as a multifocal, lymphoid, interstitial cystitis. Moreover, both $B$. Iusitaniae PotiB2 and B. burdorferi N40 induced lesions in the heart of infected mice. The lesions induced by $B$. lusitaniae PotiB2 ( 2 of 10 mice) were characterised as a severe, necrotising endarteritis of the aorta, with a minimal, mixed inflammatory infiltrate (neutrophils, macrophages and lymphoid cells) extending into the adjacent myocardium. In contrast, $B$. burgdorferi $\mathbf{N} 40$ induced a periarteritis of the pulmonary artery (7 of 10 mice), with no involvement of the endothelium and more extensive inflammation and subsequent necrosis of the adjacent myocardium. This infiltrate was composed entirely of mononuclear cells, predominantly mature lymphocytes and plasma cells. No lesions were noted in the joints or central nervous system with inoculation of strains $\mathbf{N 4 0}$ or PotiB2, and co-inoculation of either strain with Ixodes ricinus salivary gland lysate did not affect the resulting pathology. Serology, examined 8 weeks after inoculation, indicated a different reactivity in mice infected with $B$. lusitaniae PotiB2 compared with $B$. burgdorferi N40. Immunoblot analysis demonstrated that mice with lesions resulting from infection with $B$. lusitaniae PotiB2 reacted only to the flagellin protein (41 kDa) or to flagellin and OspC, whereas mice infected with B. burgdorferi $\mathrm{N} 40$ reacted with multiple high and low mol.wt proteins, including flagellin, p93, p39, OspA, OspB and OspC. These results indicate that $B$. lusitaniae PotiB2 induced pathology similar to $B$. burgdorferi $\mathrm{N} 40$ when inoculated into susceptible mice. Moreover, these results establish the first animal model of disease with $B$. lusitaniae. This mouse model can be used to characterise the immunopathogenesis of $B$. lusitaniae infection and to delineate the proteins responsible for disease induction in susceptible mice.
\end{abstract}

\section{Introduction}

Lyme borreliosis occurs world-wide and encompasses diverse symptomatology in man, including the development of erythema migrans at the tick bite site and tertiary symptoms encompassing degenerative arthritis, lesions of the central and peripheral nervous system,

Received 10 Jan. 2001; revised version received 30 March 2001; accepted 31 May 2001.

Corresponding author: Dr M. S. Núncio (e-mail: cevdi@, mail.telepac.pt). and myocarditis [1]. In Europe, the causative agents of disease in man are Borrelia burgdorferi sensu stricto and the genospecies $B$. garinii and B. afzelli $[2,3]$. The pathogenic potential of other genospecies - B. valaisiana, B. lusitaniae and B. bissettii - is currently unknown, although isolates of $B$. bissettii have been obtained recently from the cerebrospinal fluid of neuroborreliosis patients in Slovakia [4].

B. lusitaniae has been cultured from Ixodes ricinus and detected by PCR amplification in Hyalomma marginatum ticks in Portugal [5-7], Tunisia [8], Spain [9], 
Moldavia, the Ukraine and the Czech Republic [10] and Slovakia [11]. Based on comparative RFLP and sequence analysis of the $r r f-r r l$ intergenic spacer region, B. lusitaniae, specifically subspecies PotiB1, PotiB2 and PotiB3, form a clade distinct from other northern European isolates of Borrelia spp.[6].

Although cases of Lyme borreliosis have been diagnosed in Portugal [12], B. lusitaniae has not been isolated from human patients. Moreover, only one study has examined the pathogenic potential of $B$. lusitaniae in an animal model [9]. This isolate was obtained from I. ricinus ticks in Spain and, based on sequence analysis of the 16S rRNA gene, was closely related to $B$. lusitaniae PotiB2. No pathology was seen 4 weeks after needle inoculation of this isolate into the skin of $\mathrm{C} 3 \mathrm{H}$ mice [9].

The current study examined the pathogenic potential of B. lusitaniae PotiB2, isolated originally from I. ricinus ticks collected in the Alentejo region of central Portugal [7].

\section{Materials and methods}

Mice

$\mathrm{C} 3 \mathrm{H} / \mathrm{HeN}$ mice were purchased from Jackson Laboratories (Bar Harbor, ME, USA) and certified to be specific-pathogen free. All mice were housed and killed for tissue harvest and analysis according to the Animal Care and Use Committee of the Centers for Disease Control and Prevention.

Infection of mice with B. burgdorferi N4O and B. lusitaniae PotiB2 and recovery of tissues for histopathological analysis

Low passage (less than three passages in vitro) isolates of $B$. burgdorferi sensu stricto $\mathrm{N} 40$ (isolated from Westchester County, NY, USA) or B. lusitaniae PotiB2 (originally isolated from I. ricinus ticks collected in the Alentejo region of Portugal) were grown to log phase in Barbour-Stoenner-Kelly-H (BSK-H) medium (Sigma) at $33^{\circ} \mathrm{C}$ and $10^{6}$ organisms in $1 \mathrm{ml}$ of medium were inoculated subcutaneously, along the dorsal midline of mice. Mice (10 per group) were randomly assigned to receive $B$. burgdorferi N40, B. burgdorferi $\mathrm{N} 40+I$. ricinus salivary gland lysate (SGL), $B$. lusitaniae PotiB2, B. lusitaniae PotiB2 + SGL, SGL+ BSK-H or BSK-H medium alone. Four weeks after inoculation, an ear biopsy was taken and cultured to monitor individual mice for infection as described previously [13]. Eight weeks after inoculation, mice were killed and tissues (ear, bladder, heart, femorotibial joint, tibio-tarsal joint, brain and spinal cord) were placed in formaldehyde $10 \%(\mathrm{w} / \mathrm{v})$ in PBS for histological analysis.

\section{Preparation of I. ricinus salivary gland lysate for co-inoculation}

I. ricinus nymphal ticks were placed on $\mathrm{C} 3 \mathrm{H} / \mathrm{HeJ}$ mice and allowed to feed for 3 days. Ticks (50) were then collected and dissected to remove individual pairs of salivary glands. Salivary glands were then prepared for lysates (SGL) according to the method described by Labuda et al. [14]. Briefly, glands (100) were pooled, sonicated in PBS on ice (pulsed three times for $10 \mathrm{~s}$ per $40 \%$ duty cycle) (Vibra Cell Sonics Materials, Danbury, CT, USA), and centrifuged to remove all nonspecific connective tissue. The supernate was filtered through a $0.2-\mu \mathrm{m}$ filter and the total protein of the filtrate was determined by the bicinchoninic acid method according to manufacturer's instructions (Pierce, Rockford, IL, USA). A total of $25 \mu \mathrm{g}$ of SGL protein was then co-inoculated with either $B$. burgdorferi $\mathrm{N} 40$ or B. lusitaniae PotiB2 spirochaetes in a total volume of $1.0 \mathrm{ml}$ of medium.

\section{Tissue preparation for histological analysis}

Formalin-fixed tissue samples were subjected to standard processing, embedded in paraffin, and sections of 3-5 $\mu \mathrm{m}$ were prepared. All femoro-tibial and tibiotarsal joint samples were incubated for $1 \mathrm{~h}$ in rapid decalcifying solution (Shandon Corp., Pittsburgh, PA, USA) before initial sectioning. Individual tissues were then stained with haematoxylin and eosin for evaluation by light microscropy.

\section{SDS-PAGE and immunoblotting}

Low passage (less than three passages) B. lusitaniae PotiB2 strains and B. burgdorferi N40 were grown to log phase in BSK-H medium, centrifuged at 10000 rpm for $3 \mathrm{~min}$, resuspended in $1 \mathrm{ml}$ of PBS, sonicated and fractionated at $125 \mathrm{~V}$ in SDS-PAGE gels containing acrylamide $12 \% \mathrm{w} / \mathrm{v}$ (Invitrogen, Carlsbad, CA, USA) as described previously [15]. Protein profiles were obtained by silver staining (BioRad Laboratories, Hercules, CA, USA).

For immunoblotting, $10 \mu \mathrm{g}$ of $B$. lusitaniae PotiB2 or $B$. burdorferi N40 lysate were fractionated in SDS-PAGE gels containing acrylamide $10 \% \mathrm{w} / \mathrm{v}$ (Invitrogen), transferred to a nitrocellulose membrane (Invitrogen) which was blocked for $1 \mathrm{~h}$ with dried milk $10 \% \mathrm{w} / \mathrm{v}$ in $10 \mathrm{mM}$ Tris-buffered saline (TBS blotto, $\mathrm{pH}$ 8.0) [15]. The membrane was then cut into strips and incubated with a 1 in 100 dilution of mouse serum in TBS blotto overnight at $4^{\circ} \mathrm{C}$. The strips were then washed five times in TBS blotto and treated for $1 \mathrm{~h}$ with goat anti-mouse IgG and IgM alkaline phosphatase (Kirkegaard \& Perry Laboratories, Gaithersburg, MD, USA) at a 1 in 1000 dilution in TBS blotto. After washing, the strips were incubated with BCIP/NBT substrate (Kirkegaard \& Perry Laboratories) for 20 
min. Subsequent colour development was stopped by extensive washing of strips with distilled water [15].

\section{Results}

\section{Histopathological evaluation}

All mice became infected with either $B$. lusitaniae PotiB2 or B. burgdorferi $\mathrm{N} 40$ as determined by ear biopsy and culture at 28 days after inoculation of spirochaetes (data not shown). Lesions of the bladder were noted after inoculation of both $B$. burgdorferi N40 (9 of 10 mice) and B. lusitaniae PotiB2 (6 of 10 mice). Lesions were characterised as a multifocal, lymphocytic, interstitial cystitis. Multifocal, perivascular accumulations of mature lymphocytes and plasma cells were seen within the connective tissue layers below the transitional epithelium (Fig. 1a and b) at 8 weeks after inoculation of either B. burgdorferi N40 or B. lusitaniae PotiB2. These nodular accumulations of small lymphocytes and plasma cells did not extend into either the transitional epithelial layer or into the surrounding smooth muscle. Attempts to localise either $B$. lusitaniae PotiB2 or B. burgdorferi N40 spirochaetes within these nodules with a Warthin-Starry (silver) stain were unsuccessful. No quantitative or qualitative difference in these lymphoid nodules was noted in groups receiving spirochaetes in BSK-H and spirochaetes plus SGL, or when comparing the two Borrelia strains.

Lesions in the heart were also demonstrated with both Borrelia strains. B. burgdorferi N40 induced lesions in 7 of 10 inoculated mice, whereas only 2 of 10 mice inoculated with $B$. lusitaniae PotiB2 developed pathology. Moreover, differences in pathology were noted between the Borrelia isolates. In mice inoculated with $B$. burgdorferi N40, a periarteritis of the pulmonary artery was noted (Fig. 2a), with extensive inflammation and some necrosis of adjacent myocardium (Fig. 3b). In contrast, injection of B. lusitaniae PotiB2 induced single-cell necrosis of the endothelium (endarteritis) of the aorta (Fig. 2b, Fig. 3a) which then extended through the wall of the vessel into the adjacent myocardial tissue. In both cases the inflammatory infiltrates consisted of a mixture of neutrophils, lymphocytes, plasma cells and macrophages. Individual myocardial cells within areas of inflammation demonstrated vacuolar change and necrosis, especially after inoculation with $B$. burdorferi N40 (Fig. 3b). Co-inoculation of SGL with spirochaetes did not affect the extent or the number of myocardial lesions seen with either $B$. burgdorferi $\mathrm{N} 40$ or $B$. lusitaniae PotiB2.

No histopathological lesions were noted in the joints or central nervous system tissues in animals inoculated with either B. burgdorferi N40 or B. lusitaniae PotiB2, with or without SGL.

\section{Protein profiles and humoral responses}

Fig. 4 shows a comparison of the protein profiles of $B$. lusitaniae strains (lanes 2, 3 and 4) with that of the B31 strain of B. burgdorferi (lane 1) and the N40 strain of B. burgdorferi sensu stricto (lane 5). The culture of B. lusitaniae PotiB2 (lane 3) used for inoculation of mice did not express outer-surface protein A (OspA), nor did the other Portuguese isolates of $B$. lusitaniae, PotiB1 (lane 2) and PotiB3 (lane 4). Bands representing the outer-surface protein B (OspB), and the flagellin protein (Fla) were noted in each Poti strain as well as the B. burgdorferi N40 strain used for in-vivo studies.

The serological response to both B. burgdorferi N40 and $B$. lusitaniae PotiB2 was analysed at 8 weeks after inoculation of spirochaetes. As seen in the immunoblots in Fig. 5, reactivity to B. lusitaniae PotiB2 in all mice was restricted to the flagellin protein or outersurface protein $\mathrm{C}(\mathrm{OspC})$, or both. Each of these mice had lesions in both the bladder and the heart. Reactions between reference monoclonal antibodies directed toward flagellin, OspA, OspB and OspC and $B$. lusitaniae PotiB2 indicated that the bands in the 41and $23-\mathrm{kDa}$ regions corresponded to flagellin and OspC (data not shown). The humoral response to $B$. burgdorferi $\mathrm{N} 40$ at 8 weeks after inoculation included reactivity to a range of large and small mol. wt proteins, including OspA, OspB, OspC and flagellin protein (Fig. 5). As with B. lusitaniae PotiB2, only representative serum samples, from animals that developed pathology of both the heart or bladder, are presented in Fig. 5.

\section{Discussion}

To our knowledge this is the first report of the pathogenic potential of $B$. lusitaniae in mice. This study indicated that at least one strain of $B$. lusitaniae, PotiB2, isolated originally from $I$. ricinus ticks in southern Portugal [7], induced both nodular interstitial cystitis and vasculitis of the great vessels of the heart. This contrasts with an earlier unsuccessful attempt to induce pathology by needle inoculation of a Spanish isolate of B. lusitaniae (PV8) in $\mathrm{C} 3 \mathrm{H}$ mice [9]. The reasons for the differences between these studies are varied and include inoculum size $\left(10^{4}\right.$ spirochaetes of B. lusitaniae PV8; $10^{6}$ of B. lusitaniae PotiB2) and interval of time to demonstrate dissemination from the skin and resultant pathology of internal organs (4 weeks for B. lusitaniae PV8; 8 weeks for B. lusitaniae PotiB2). Although phylogenetic analysis revealed homology between the $16 \mathrm{~S}$ genes of $B$. lusitaniae PV8 and PotiB2, the current study may indicate a difference in virulence between these two isolates.

In the present study, a higher percentage of mice infected with B. burgdorferi N40 developed lesions of the bladder and the heart, although the severity of inflammation was not qualitatively different to that seen 

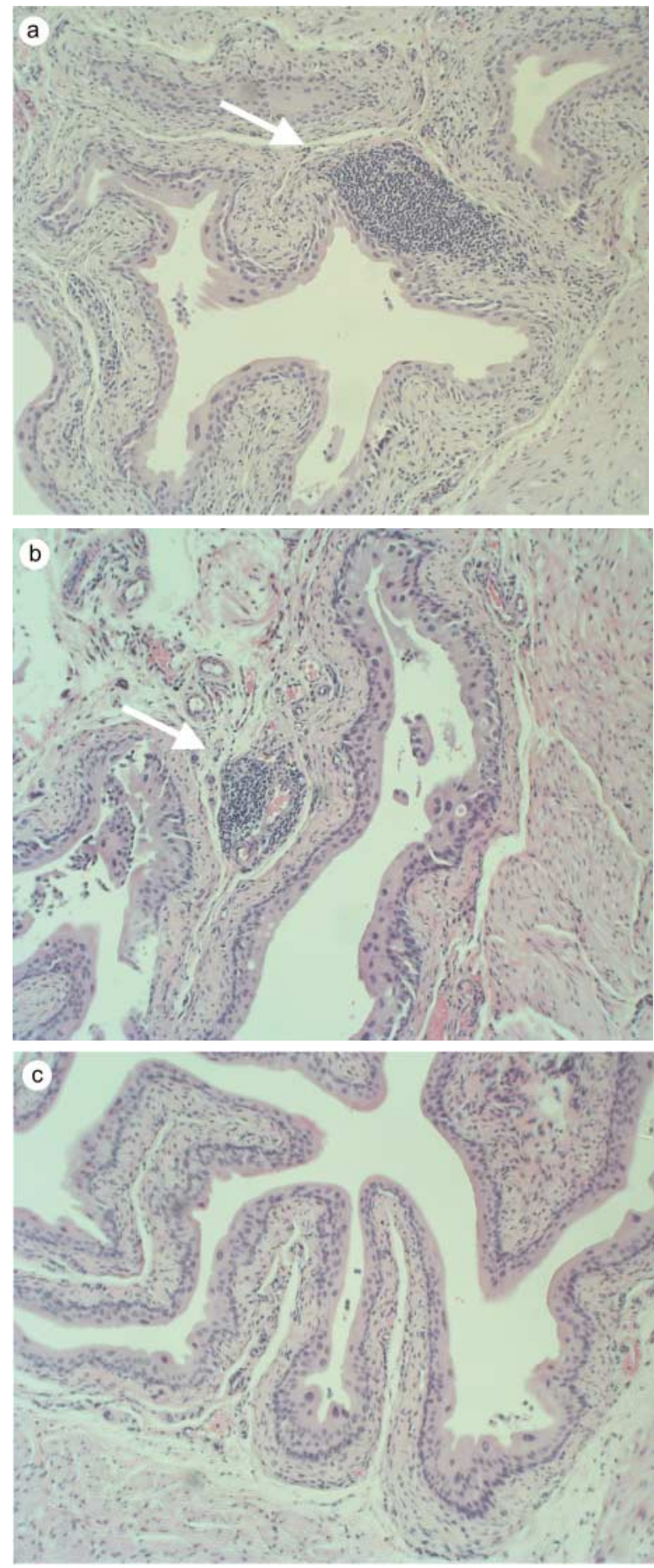

Fig. 1. Histopathological sections of bladder, 8 weeks after injection of spirochaetes (H\&E 20×). a, B. burgdorferi N40; b, B. lusitaniae PotiB2; c, sham-inoculated control mouse. Arrows indicate perivascular lymphoid nodules within the interstitium.

with B. lusitaniae PotiB2. Interestingly, although the lesions in the bladder were similar histologically, lesions in the heart were noticeably different. $B$. lusitaniae PotiB2 induced an inflammatory response of the endothelium, with evidence of single-cell necrosis of endothelial cells, which occurred solely within the aorta. In contrast, B. burgdorferi N40 induced pathology within the pulmonary artery, characterised as
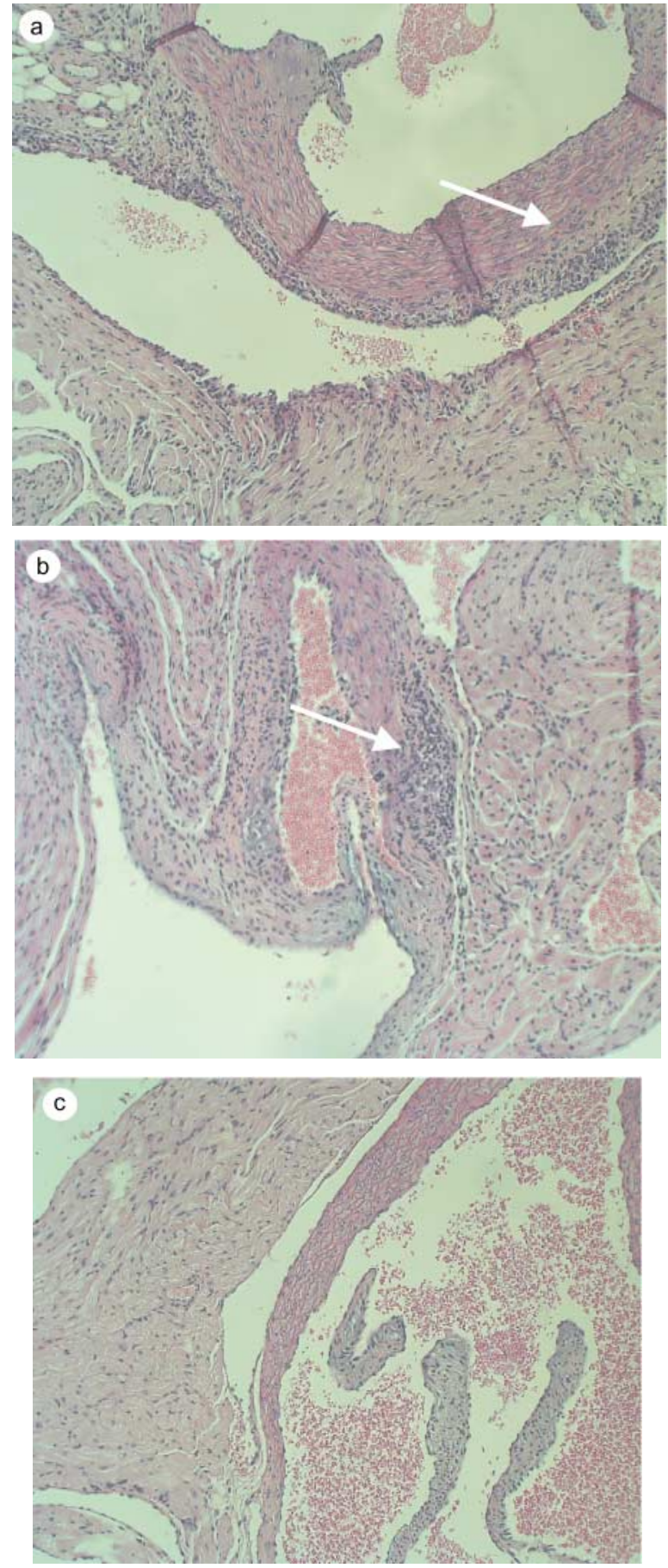

Fig. 2. Histopathological sections of heart, 8 weeks after injection of spirochaetes (H\&E $20 \times)$. a, arrow indicates perivasculitis in the wall of the pulmonary artery after inoculation with $B$. burgdorferi $\mathrm{N} 40 ; \mathbf{b}$, arrow indicates vasculitis of the aorta after inoculation with $B$. lusitaniae PotiB2; $\mathbf{c}$, aorta of a shaminoculated control mouse.

an inflammation of the lateral portion of the vascular tunic, which extended laterally causing necrosis of adjacent myocardium. A similar vasculitis of the great vessels has been demonstrated previously with needle inoculation of B. burgdorferi N40 [16]. Whether this difference in tissue tropism and resultant inflammatory pattern between $B$. burgdorferi $\mathrm{N} 40$ and B. lusitaniae PotiB2 is due to a difference in outer-surface protein expression of cultured spirochaetes remains to be 

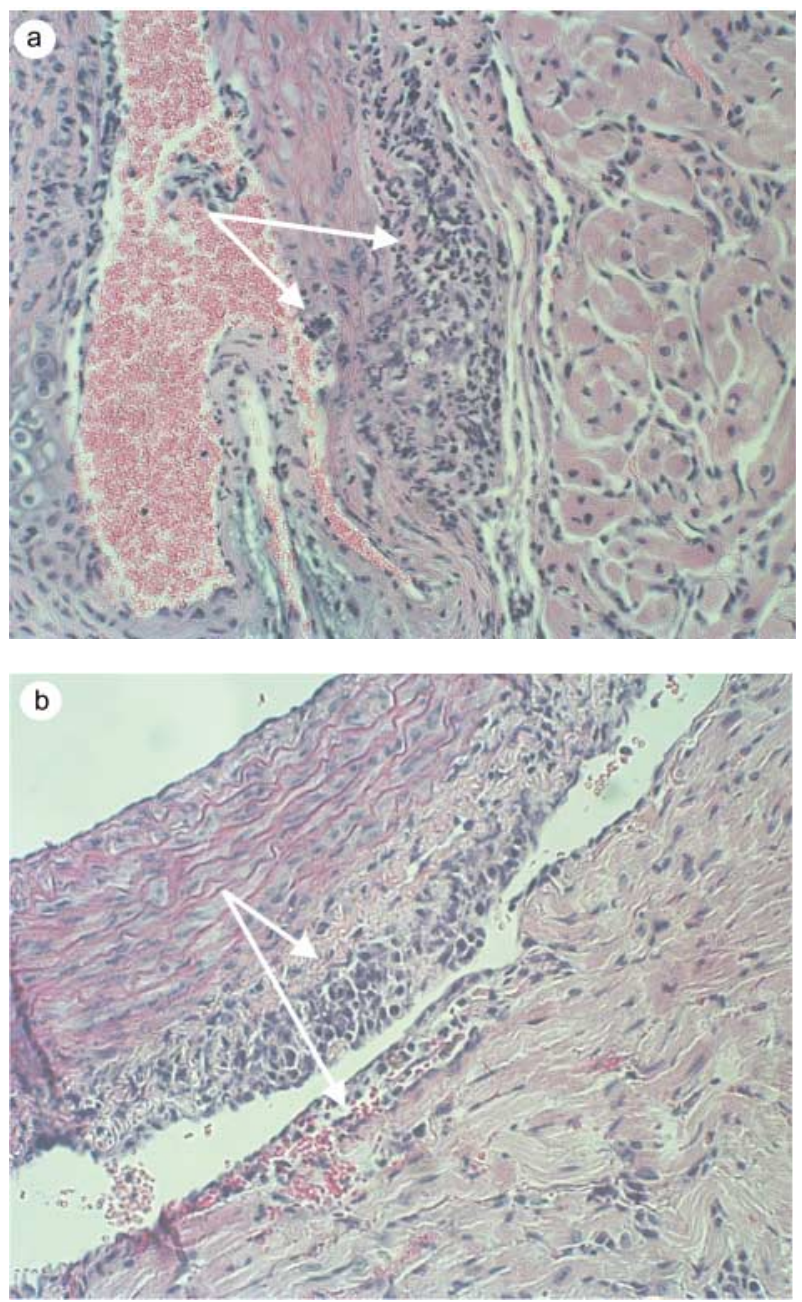

Fig. 3. Histopathological sections of heart, 8 weeks after injection of spirochaetes $(H \& E 40 \times)$. a, arrows indicate endarteritis of the aorta which extends through the vascular wall and into the adjacent myocardium, after inoculation with $B$. lusitaniae PotiB2; $\mathbf{b}$, arrows indicate periarteritis of the pulmonary artery extending into the surrounding myocardium after inoculation with B. burgdorferi $\mathrm{N} 40$.

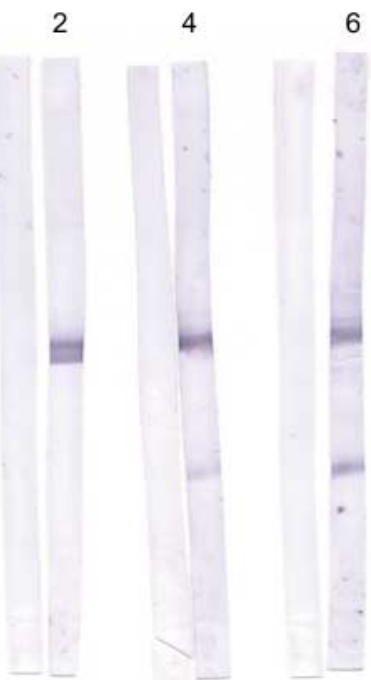

PATHOLOGY OF BORRELIA LUSITANIAE INFECTION

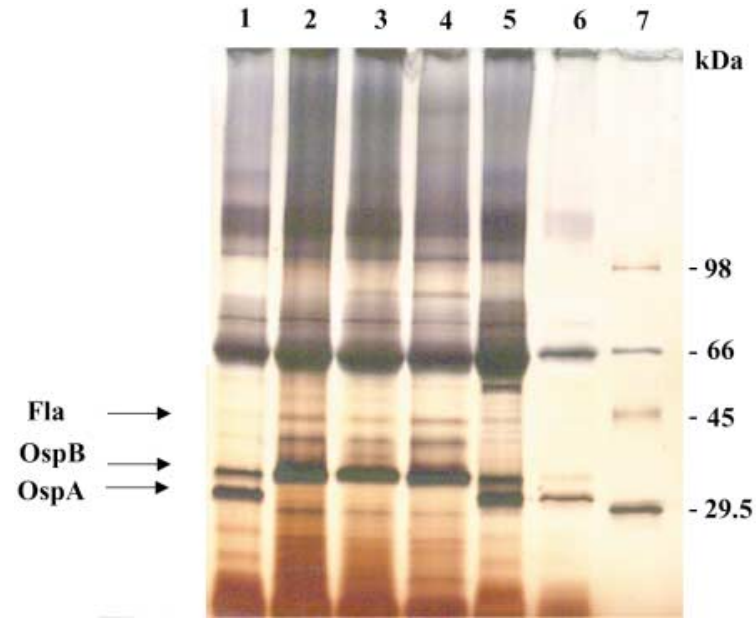

Fig. 4. Protein profile of Portuguese isolates of B. lusitaniae compared to B. burgdorferi strains B31 and N40. Low-passage isolates were fractionated by SDS-PAGE and stained with silver. Lane 1, B. burgdorferi $\mathrm{B} 31 ; 2, B$. lusitaniae strain PotiB1; 3, B. lusitaniae strain PotiB2; 4, B. lusitaniae strain PotiB3; 5, B. burgdorferi strain N40; 6 and 7, mol. wt standards.

investigated. Also, the co-inoculation of I. ricinus SGL with B. lusitaniae PotiB2 or B. burgdorferi N40 did not affect the extent of pathology described here. Whether this was due to the preparation of SGL inoculum, the amount of SGL inoculated, or the time point examined after spirochaete inoculation remains to be tested. Moreover, as noted earlier, neither $B$. lusitaniae PotiB2 nor B. burgdorferi N40 induced joint lesions. It is possible that in the examination of mice at 8 weeks after inoculation, the acute or chronic time point for evaluation of joint disease was missed. Earlier reports indicated that $B$. burgdorferi $\mathrm{N} 40$ induced an acute joint lesion at 3-4 weeks after spirochaete inoculation which had resolved by day 90 [16]. Studies in this laboratory have demonstrated joint pathology at b

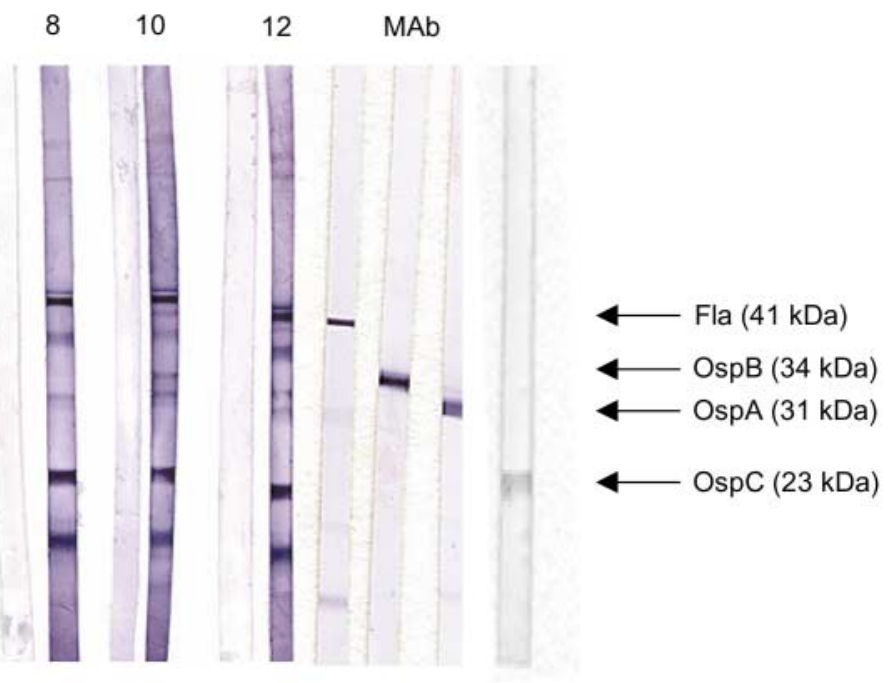

Fig. 5. Humoral responses to $B$. lusitaniae PotiB2 (a) and B. burgdorferi $\mathrm{N} 40$ (b) after needle inoculation with spirochaetes. Lanes $\mathbf{2}, \mathbf{4}$ and $\mathbf{6}$, reaction between individual sera from mice infected with $B$. lusitaniae and cultured $B$. lusitaniae PotiB2; 8, 10, and 12, reaction between individual sera from mice infected with $B$. burgdorferi $\mathrm{N} 40$ and the cultured $B$. burgdorferi spirochaetes; 1, 3, 5, 7, 9 and 11 (not labelled), represent pre-infection sera of individual mice. MAb, reaction between reference monoclonal antibodies directed toward flagellin, OspB, OspA and OspC and B. burgdorferi N40. 
12 weeks after inoculation of B. burgdorferi N40 by nymphal ticks which persisted to 18 weeks [17].

As demonstrated here, B. lusitaniae PotiB2 cultured in vitro does not express OspA, and animals infected with this organism produce an antibody response to only the flagellin and OspC proteins at 8 weeks after inoculation. Moreover, at least one animal, which had lesions of both bladder and heart, demonstrated an antibody response that reacted with only the flagellin protein. In contrast, those animals infected with $B$. burgdorferi $\mathrm{N} 40$ produced an antibody response to several proteins, including OspA, OspB, OspC and flagellin. Further studies are needed to properly characterise both the humoral and T-cell responses to B. lusitaniae PotiB2 and to correlate these responses with resultant tissue pathology. What is apparent from this study is that pathology induced by $B$. lusitaniae PotiB2 in the bladder and heart is not dependent on either the humoral or T-cell response to OspA. However, this needs to be tested in a rigorous manner by the natural route of transmission by using I. ricinus nymphal ticks.

Since the first case of Lyme borreliosis was reported in Portugal in 1989 [18], all strains of Borrelia isolated from ticks in southern Portugal have been classified within a distinct clade of Poti strains of $B$. lusitaniae $[5,6,19]$. To date, no human isolates have been investigated in an animal model. The establishment of the pathogenic potential of $B$. lusitaniae PotiB2 in a mouse model affords the opportunity to investigate the pathogenesis of this unique isolate which lacks expression of OspA. Future studies will include the inoculation of $B$. lusitaniae PotiB2 by tick bite, a more detailed examination of outer-surface protein expression in affected tissues, and an examination of the adaptive immune responses that correlate with lesion development in this animal model.

We thank histotechnologists Ana Cristina de Encarnação Fernandes and Ana Alexandra Cabrita Maria, São Bernardo Hospital, Setúbal, Portugal, for expert technical assistance in preparing histological sections.

\section{References}

1. Dennis DT. Lyme disease. Dermatol Clin 1995; 13: 537-551. 2. Wang G, van Dam AP, Schwartz I, Dankert J. Molecular typing of Borrelia burgdorferi sensu lato: taxonomic, epidemiological, and clinical implications. Clin Microbiol Rev 1999; 12: 633-653.

3. Van Dam AP, Kuiper H, Vos K et al. Different genospecies of Borrelia burgdorferi are associated with distinct clinical manifestations of Lyme borreliosis. Clin Infect Dis 1993; 17: $708-717$.

4. Strle F, Picken RN, Cheng $\mathrm{Y}$ et al. Clinical findings for patients with Lyme borreliosis caused by Borrelia burgdorferi sensu lato with genotypic and phenotypic similarities to strain 25015. Clin Infect Dis 1997; 25: 273-280.

5. La Fleche A, Postic D, Girardet K, Peter O, Baranton G. Characterization of Borrelia lusitaniae sp. nov. by $16 \mathrm{~S}$ ribosomal DNA sequence analysis. Int J Syst Bacteriol 1997; 47: $921-925$.

6. De Michelis S, Sewell H-S, Collares-Pereira M et al. Genetic diversity of Borrelia burgdorferi sensu lato in ticks from mainland Portugal. J Clin Microbiol 2000; 38: 2128-2133.

7. Núncio MS, Péter O, Alves MJ, Bacellar F, Filipe AR. Isolamento e caracterização de borrélias de Ixodes ricinus L. em Portugal. Rev Port Doenç Infec 1993; 16: 175-179.

8. Zhioua E, Bouattour A, Hu CM et al. Infections of Ixodes ricinus (Acari:Ixodidae) by Borrelia burgdorferi sensu lato in North Africa. J Med Entomol 1999; 36: 216-218.

9. Escudero R, Barral M, Pérez A et al. Molecular and pathogenic characterization of Borrelia burgdorferi sensu lato isolates from Spain. J Clin Microbiol 2000; 38: 4026-4033.

10. Postic D, Korenberg E, Gorelova N, Kovalevski YV, Bellenger E, Baranton G. Borrelia burgdorferi sensu lato in Russia and neighboring countries: high incidence of mixed isolates. Res Microbiol 1997; 148: 691-702.

11. Gern L, Hu CM, Kocianova E, Vyrostekova V, Rehacek J. Genetic diversity of Borrelia burgdorferi sensu lato isolates obtained from Ixodes ricinus collected in Slovakia. Eur $J$ Epidemiol 1999; 15: 665-669.

12. Morais JA, Abranches J, Parra J et al. Artrite de Lyme em Portugal. A proposito dos primeiros casos diagnosticados em Portugal. Rev Port Doenç Infec 1994; 17: 183-195.

13. Sinsky RJ, Piesman J. Ear punch biopsy method for detection and isolation of Borrelia burgdorferi from rodents. J Clin Microbiol 1989; 27: 1723-1727.

14. Labuda M, Jones LD, Williams T, Nuttall PA. Enhancement of tick-borne encephalitis virus transmission by tick salivary gland extracts. Med Vet Entomol 1993; 7: 193-196.

15. Zeidner N, Dreitz M, Belasco D, Fish D. Suppression of acute Ixodes scapularis-induced Borrelia burgdorferi infection using tumor necrosis factor- $\alpha$, interleukin-2, and interferon- $\gamma$. J Infect Dis 1996; 173: 187-195.

16. Barthold SW, de Souza MS, Janotka JL, Smith AL, Persing DH. Chronic Lyme borreliosis in the laboratory mouse. Am J Pathol 1993; 143: 959-971.

17. Zeidner NS, Schneider BS, Dolan MC, Piesman J. An analysis of spirochete load, strain and pathology in a tick-transmitted model of lyme borreliosis. J Zoon Vec Borne Dis 2001; 1: 33-42.

18. Morais JA, Filipe AR, Núncio MS. Doença de Lyme em Portugal - caso clinico. Rev Port Doenç Infec 1989; 12: 261-276.

19. Postic D, Assous MV, Grimont PAD, Baranton G. Diversity of Borrelia burgdorferi sensu lato evidenced by restriction fragment length polymorphism of $\operatorname{rrf}(5 \mathrm{~S})-\operatorname{rrl}(23 \mathrm{~S})$ intergenic spacer amplicons. Int J Syst Bacteriol 1994; 44: 743-752. 